\title{
Primary combined small and squamous cell carcinoma of the hypopharynx: A case report
}

\author{
KIYOSHI MISAWA ${ }^{1}$, HIDEYA KAWASAKI ${ }^{2}$, SHIORI ENDO ${ }^{1}$, DAIKI MOCHIZUKI ${ }^{1}$, \\ KOTARO MORITA ${ }^{1}$, YUICHI HASHIMOTO ${ }^{1}$, YUKI MISAWA ${ }^{1}$, HIROTOSHI KIKUCHI ${ }^{3}$, \\ TAKEHARU KANAZAWA ${ }^{4}$, TOSHIHIDE IWASHITA ${ }^{2}$ and HIROYUKI MINETA ${ }^{1}$ \\ ${ }^{1}$ Department of Otolaryngology/Head and Neck Surgery; ${ }^{2}$ Department of Regenerative and Infectious Pathology; \\ ${ }^{3}$ Second Department of Surgery, Hamamatsu University School of Medicine, Hamamatsu, Shizuoka 431-3192; \\ ${ }^{4}$ Department of Otolaryngology/Head and Neck Surgery, Jichi Medical University, Shimotsuke, Tochigi 329-0498, Japan
}

Received September 1, 2015; Accepted January 29, 2016

DOI: $10.3892 / \mathrm{mco} .2016 .788$

\begin{abstract}
We herein report a very rare case of combined small-cell carcinoma ( $\mathrm{SmCC}$ ) of the hypopharynx, with a squamous cell carcinoma (SqCC) element. A 74-year-old man presented with a 3-month history of throat pain and hoarseness. On hypopharyngoscopy, a tumor was identified in the right anterior wall of the piriform sinus and, following examination of a biopsy sample, the lesion was diagnosed as SqCC. Total laryngectomy with bilateral neck dissection was performed and the malignancy was diagnosed as combined SmCC. One month after surgery, concomitant chemoradiotherapy with cisplatin and etoposide was administered. Immunohistochemically, the SmCC element was positive for CD56 and Ki-67 (50.2\%), whereas the SqCC element was positive for cytokeratin $34 \beta \mathrm{E} 12$ and Ki-67 (47.5\%). Furthermore, the SmCC element was positive for KIT and platelet-derived growth factor $\alpha$ (PDGFR $\alpha)$, while the SqCC element was positive for epidermal growth factor receptor (EGFR) and PDGFR $\alpha$. By genetic analysis, a silent mutation in the PDGFR $\alpha$ gene was recognized. The expression of KIT, PDGFR $\alpha$ and EGFR in this case provided evidence that combined SmCC may be a candidate for molecular-targeted therapy, although further investigations are required.
\end{abstract}

\section{Introduction}

Primary malignant tumors of the hypopharynx are predominantly squamous cell carcinomas (SqCCs), as primary small-cell carcinoma ( $\mathrm{SmCC}$ ) of the hypopharynx is rare. Combined primary SmCC and SqCC of the hypopharynx,

Correspondence to: Dr Kiyoshi Misawa, Department of Otolaryngology/Head and Neck Surgery, Hamamatsu University School of Medicine, 1-20-1 Handayama, Hamamatsu, Shizuoka 431-3192, Japan

E-mail: kiyoshim@hama-med.ac.jp

Key words: combined small and squamous cell carcinoma, hypopharynx, KIT, platelet-derived growth factor receptor $\alpha$ referred to as composite tumor of the hypopharynx, is even more rare, with only 3 cases previously reported (1-3). Therefore, there is little information available on the optimal management of these patients. We herein report a the case of a patient with primary combined SmCC with an SqCC element and investigate the expression of specific proteins for molecular-targeted therapy.

\section{Case report}

A 74-year-old man, with a $~ 50$-year history of excessive alcohol consumption and smoking, presented with a 3-month history of throat pain and hoarseness. There was no history of weight loss, dysphagia, or dyspnea. On hypopharyngoscopy, a tumor was identified in the right anterior wall of the piriform sinus (Fig. 1A). A pathologist analyzed the biopsy sample and diagnosed the lesion as SqCC, as no SmCC component was identified in the biopsy specimen. The right vocal cord was not fixed. A contrast computed tomography (CT) scan of the neck revealed a heterogeneously enhanced tumor sized $25 \times 17 \times 38 \mathrm{~mm}$ extending through the right piriform sinus (Fig. 1B). Moreover, fluorodeoxyglucose positron emission tomography (FDG-PET) revealed high-level accumulation in the primary tumor, with a maximum standardized uptake value of 14.6. There was no evidence of cervical lymph node metastasis, primary lung tumor, or distant metastasis. The patient was diagnosed with hypopharyngeal cancer classified as T2N0M0, according to the 2009 Union for International Cancer Control staging system (4). We performed a tracheostomy, total laryngectomy, right hemithyroidectomy and bilateral lateral neck dissection (level II-IV). The macroscopic surgical specimen is presented in Fig. 1C. The specimen consisted of the resected larynx and right piriform sinus tumor. A 25x20-mm deeply ulcerated tumor was found in the right piriform sinus. Microscopically, two components were identified in the lesion, namely a poorly differentiated SqCC and an SmCC containing chromatin-rich nuclei with scanty cytoplasm (Fig. 2A). Pathologically, multiple metastatic cervical lymph nodes were identifed bilaterally (right side, levels II and III; and left side, level III). Although the surgical margin was narrow, it was negative for cancer cells. 
Concomitant chemoradiotherapy (cisplatin + etoposide with $45 \mathrm{~Gy}$ ) was administered postoperatively. However, after the first course, adjuvant chemotherapy was discontinued due to prolonged bone marrow suppression, although the therapy was effective. However, despite treatment, distant metastases in the thoracic vertebrae (T5, 9 and 10) were confirmed by CT 6 months later. The patient's condition started to deteriorate and he succumbed to the disease 7 months after the initial treatment.

Pathology. On gross examination of the laryngectomy specimen, a mass involving the right piriform sinus was palpable. The tumor extended superiorly to involve the right supraglottis and exhibited variable gross morphology, ranging from ulcerated, to nodular, to plaque-like growth. The tumor infiltrated $1.3 \mathrm{~cm}$ in depth, but did not involve the thyroid cartilage, vocal cord, or trachea. Microscopic sections from the larynx revealed a combined tumor, composed predominantly of $\mathrm{SmCC}$ localized in the upper side and SqCC localized in the lower side (Figs. 1 and 2A). We observed 2 components in the lesion: A poorly differentiated SqCC and a small-sized SmCC with finely granular, hyperchromatic nuclei, inconspicuous nucleoli and scanty cytoplasm. There was a transitional area between the SmCC and SqCC (Fig. 2A and B). The metastatic potential of the lesion was further supported by the SmCC component found to infiltrate lymphatic vessels (Fig. 2C). The metastatic lymph nodes primarily involved the SmCC component (Fig. 2D).

Immunohistochemically, the SmCC strongly expressed CD56 (Fig. 2E) and cytokeratin (CK) AE1/AE3. Diffuse CD56 positivity in the SmCC component in the cervical lymph nodes supported the histological diagnosis (Fig. 2F). The SmCC component was also negative for CK 34ßE12 (Fig. 2G), p63, synaptophysin, chromogranin A and CD45. The SqCC component was negative for neuroendocrine markers, including CD56, synaptophysin and chromogranin A, although it was positive for CK 34ßE12 (Fig. 2H). Immunoreactivity to Ki-67 was seen in the nuclei of tumor cells. In the SmCC component, the Ki-67 labeling index was 50.2\%, while that in the SqCC component was $47.5 \%$ (Fig. 2I and J).

To predict the prognosis of this patient, we evaluated the expression of epidermal growth factor receptor (EGFR). Furthermore, to evaluate the possibility of using molecular-targeted therapy for combined SmCC and SqCC, the expression of proteins such as platelet-derived growth factor receptor $\alpha$ (PDGFR $\alpha$ ), vascular endothelial growth factor receptor 2 (VEGFR2) and KIT was examined. On immunohistochemical analysis, the SmCC element was strongly positive for KIT and PDGFR $\alpha$; however, EGFR and VEGFR2 were not expressed (Fig. 3A, C and E). The SqCC element was mildly positive for PDGFR $\alpha$ and EGFR; however, KIT and VEGFR2 were not expressed (Fig. 3B, D and F).

DNA extraction, genetic analysis and human papillomavirus $(H P V)$ polymerase chain reaction. DNA was isolated from the SmCC element in specimens obtained during surgery. Genomic DNA was extracted from frozen tumor specimens using the QIAamp DNA Mini Kit (Qiagen, Hilden, Germany), according to the manufacturer's protocol.

A molecular genetic analysis of $K I T$ (exons 9, 11, 13 and 17) and PDGFR $\alpha$ (exons 12, 14 and 18) was performed using the

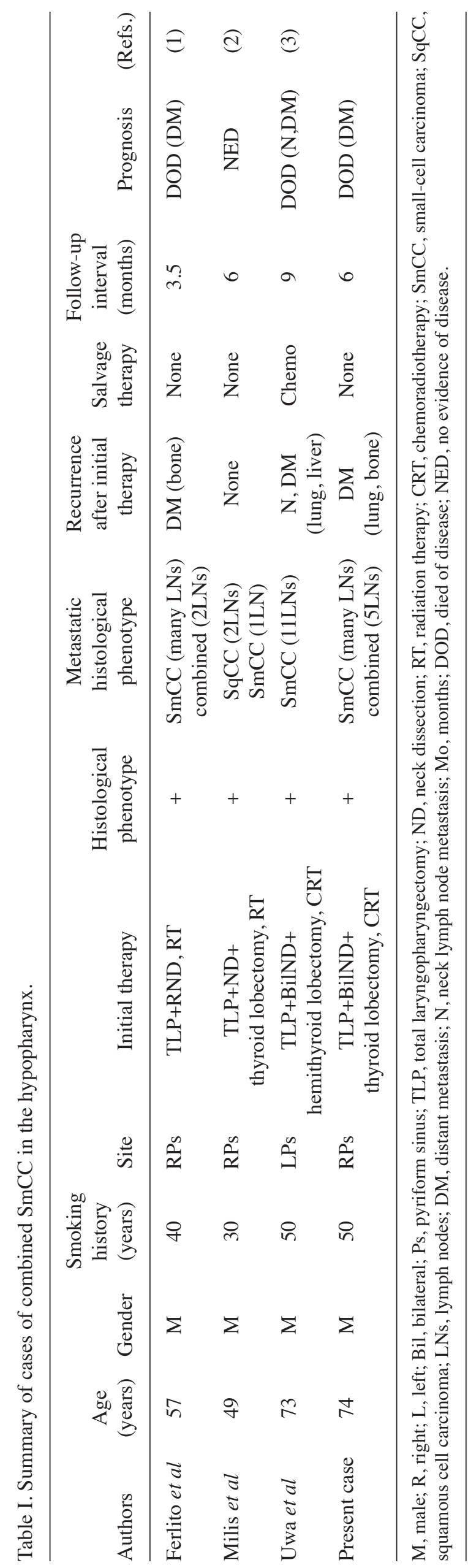


A

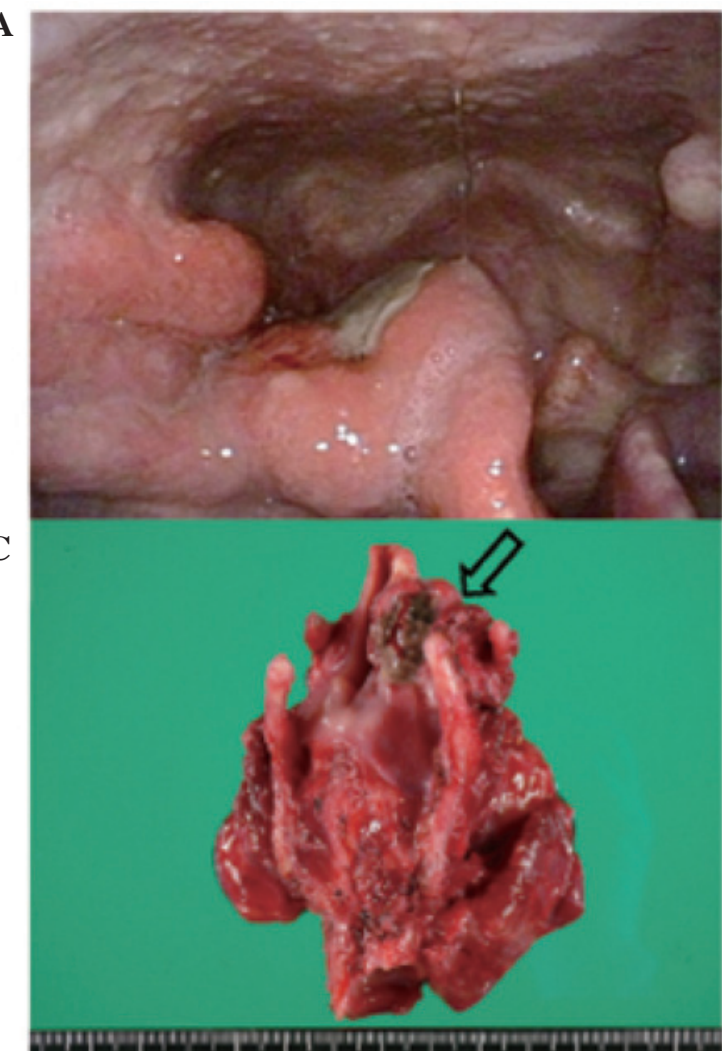

B

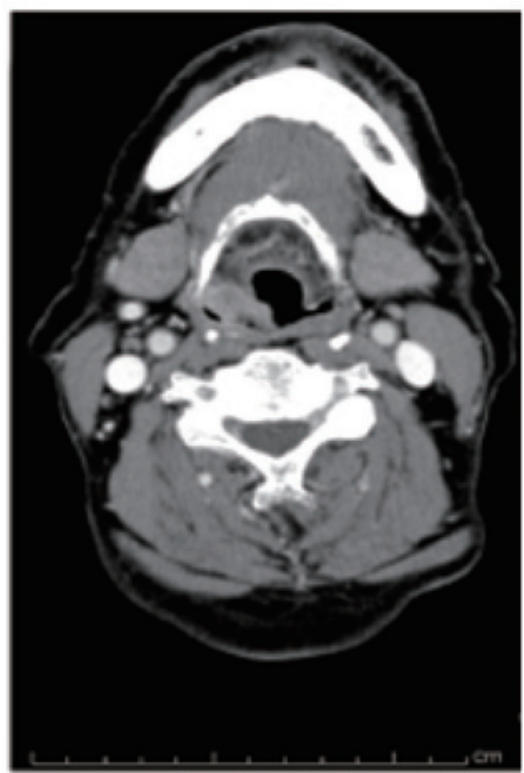

Figure 1. (A) Hypopharyngeal fiberscopic image. An ulcerated tumor was identified in the right piriform sinus. (B) Contrast computed tomography scan of the neck prior to treatment. An enhanced tumor was identified in the right piriform sinus. (C) Macroscopic surgical specimen. A deeply ulcerated tumor is seen in the right piriform sinus (arrow).

polymerase chain reaction (PCR) direct sequencing method, as previously reported (5-8). PCR products were extracted and subjected to a computed automatic DNA sequencing (ABI PRISM 3100 Genetic Analyzer; Applied Biosystems, Carlsbad, CA, USA). This case exhibited a silent mutation in exon 12 of PDGFR (Fig. 4); we observed an $\mathrm{A} \rightarrow \mathrm{G}$ change in codon 567 $(\mathrm{CCA} \rightarrow \mathrm{CCG})$. Analysis of exon 12 of $P D G F R \alpha$ from other normal tonsillar tissues revealed a similar $A \rightarrow G$ change at the 1849 nucleotide position. Comparison of this sequence variation with the single-nucleotide polymorphism (SNP) database revealed the presence of a known SNP at codon 567 of PDGFR (9).

The HPV status was determined using the HPV Typing Set (Takara Bio., Tokyo, Japan), a PCR primer set specifically designed to identify HPV genotypes $16,18,31,33,35,52$ and 58 in genomic DNA. The PCR HPV Typing Set method was used according to the manufacturer's protocol. The PCR products were separated by electrophoresis through a $9 \%$ polyacrylamide gel and stained with ethidium bromide. Our case was negative and not considered as a high-risk HPV status (Fig. 5).

\section{Discussion}

In the head and neck, SmCC most commonly arises in the larynx, but has also been reported in the sinonasal tract and salivary glands $(10,11)$. Combined SmCC of the larynx has only been reported in 17 cases in the literature to date. The majority of these patients were men and in the 6th-7th decade of life. Moreover, the majority of the patients succumbed to the disease within 2 years of diagnosis, even with adjuvant radiation and chemotherapy (12-14). Despite the rarity of these tumors, the clinical behavior of combined SmCC of the larynx appears to be similar to that of the hypopharynx. Metastasis from a primary lung SmCC must be carefully distinguished from a primary hypopharyngeal or laryngeal SmCC by imaging studies of the lung (15). In this case, FDG-PET revealed high-level accumulation in the hypopharynx, without evidence of a lung tumor or distant metastasis. A chest contrast CT scan confirmed the absence of a primary lung tumor.

According to the World Health Organization classification of head and neck tumors in 2005, neuroendocrine carcinomas may be classified as typical carcinoid, atypical carcinoid, and SmCC (16). Neuroendocrine types of SmCC associated with an $\mathrm{SqCC}$ component are referred to as combined carcinomas. They are unusual, representing $<10 \%$ of all neuroendocrine types (17). Neuroendocrine neoplasms of the hypopharynx and larynx constitute a morphologicaly heterogeneous group of tumors, with considerable differences in clinical behavior and very different treatment strategies (12). Combined SmCC of the hypopharynx is extremely rare and is often difficult to pathologically diagnose prior to surgical treatment. Moreover, the prognosis is very poor due to early metastasis, without established treatment regimen (3). Of the 4 reported cases of combined SmCC of the hypopharynx, including our case, 3 succumbed to the disease within 1 year (Table I). Multi-modality therapy should be performed for combined SmCC of the head and neck region. Therefore, new therapeutic 

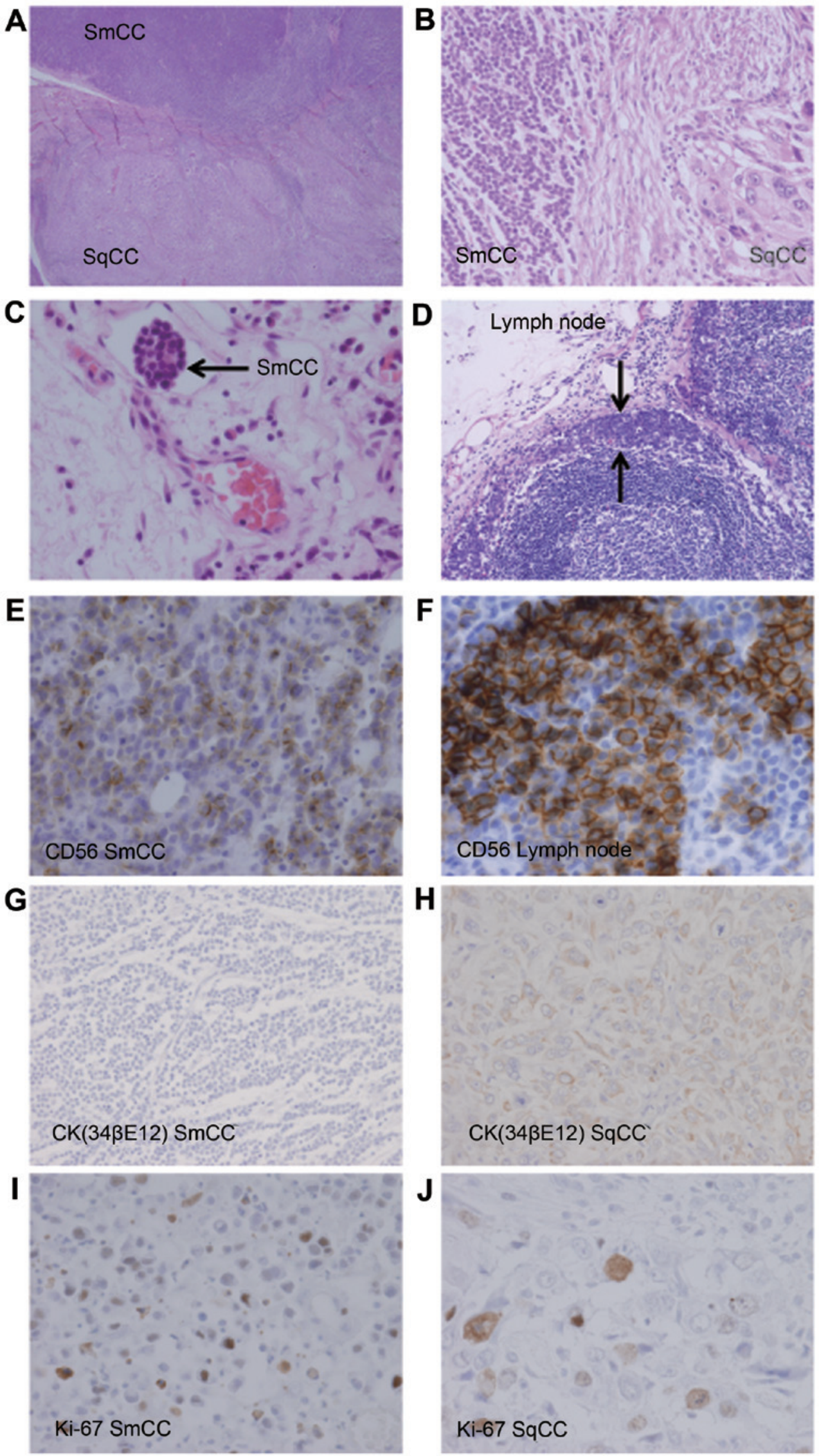

Figure 2. Microscopic images of the surgical specimen. (A) Hematoxylin and eosin staining. This tumor consisted of a combination of small-cell carcinoma (SmCC) and squamous cell carcinoma (SqCC). (B) Collision phase showing invasive SqCC in gradual transition with adjacent SmCC. (C) SmCC component (arrow) infiltrating a lymphatic vessel. (D) Metastatic lymph nodes mainly involved the SmCC component (arrows). (E) CD56 was expressed in the SmCC component. (F) The SmCC component of the neck lymph node was positive for CD56. (G) The SmCC component was negative for cytokeratin (CK) 34ßE12. (H) The SqCC component was positive for CK 34ßE12. (I) Staining of the SmCC component revealed a high Ki-67 labeling index of 50.2\%. (J) The Ki-67 labeling index was $47.5 \%$ in the SqCC component. 

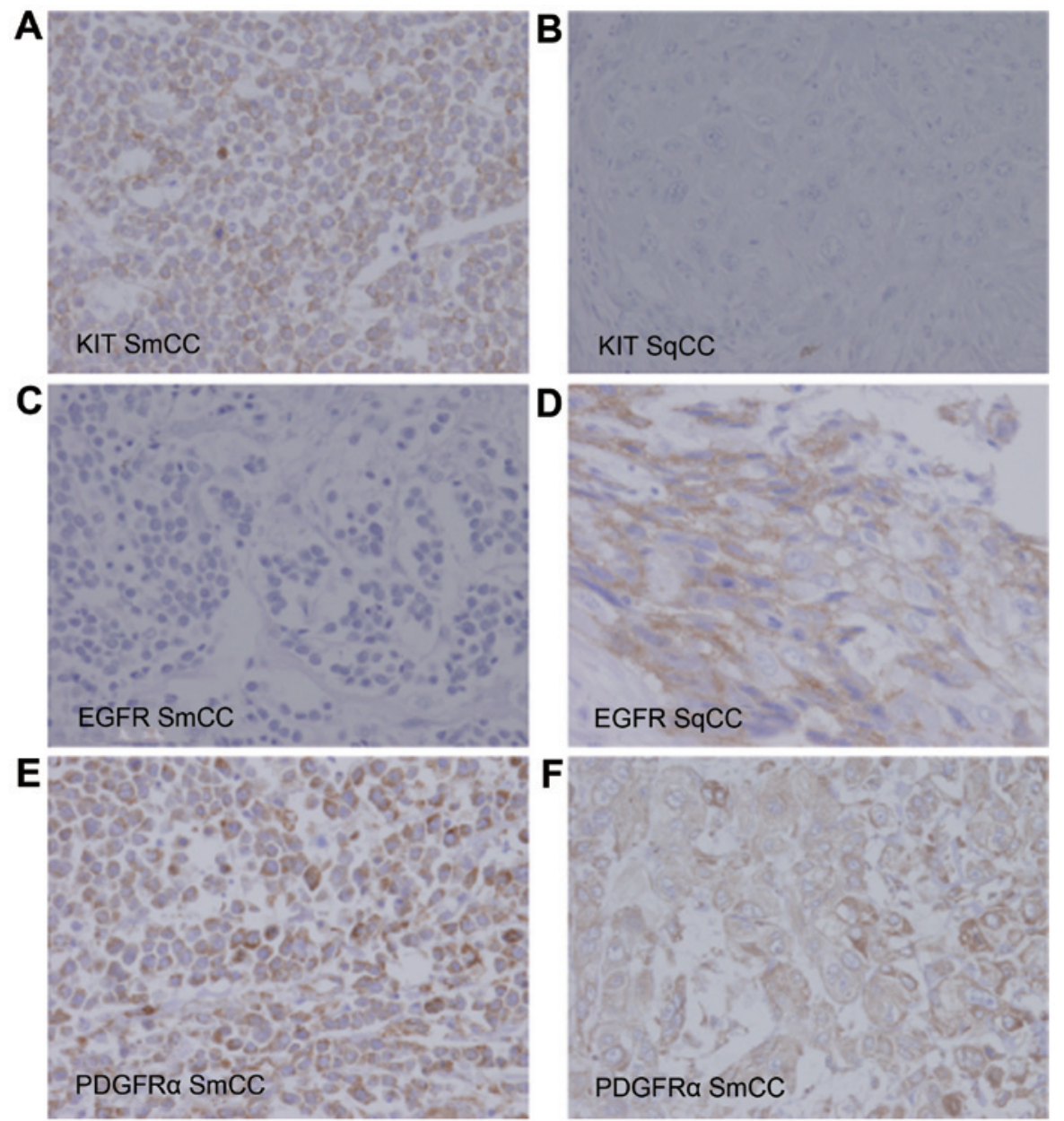

Figure 3. (A) KIT was expressed in the small-cell carcinoma (SmCC) component, but (B) not in the squamous cell carcinoma (SqCC) component. (C) The SmCC component was negative for epidermal growth factor receptor (EGFR), but (D) the SqCC component was positive for EGFR. (E and F) Immunostaining demonstrated that both components were positive for platelet-derived growth factor receptor $\alpha$ (PDGFR $\alpha$ ).

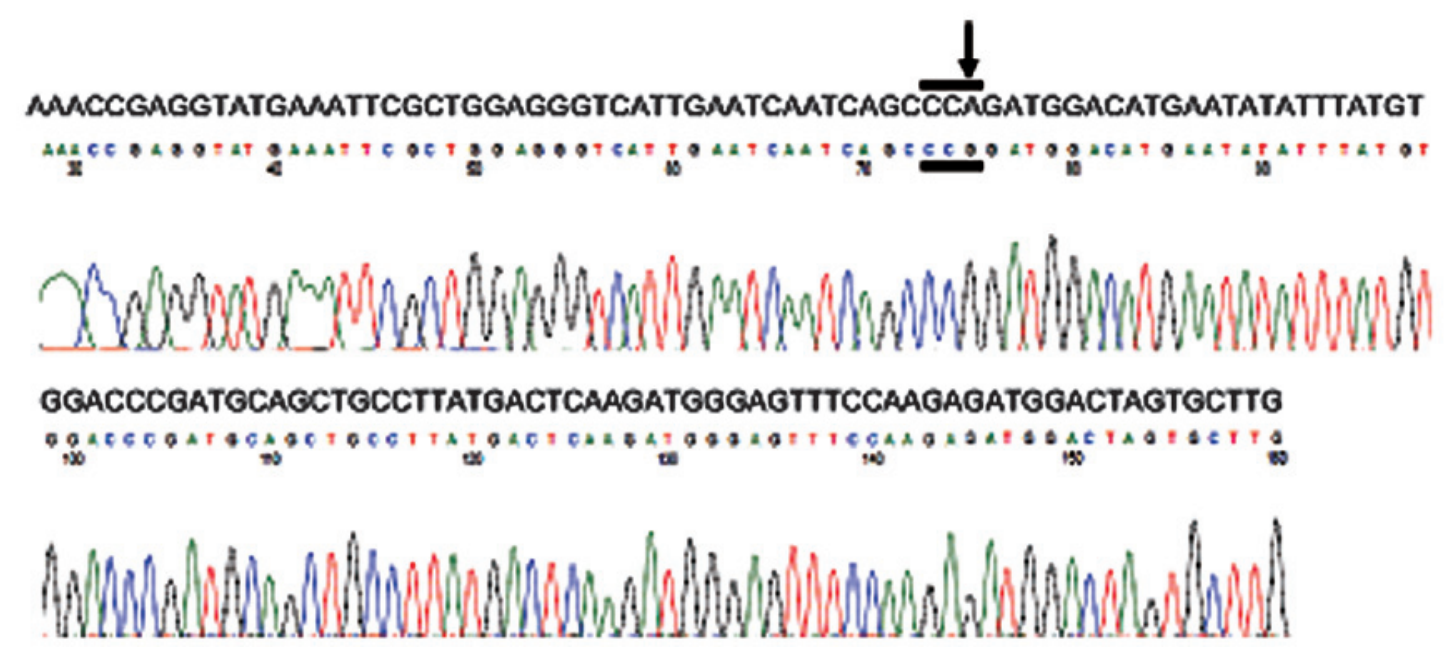

Figure 4. Electropherogram with relative sequence for the small-cell carcinoma elements of exon 12 of the platelet-derived growth factor receptor $\alpha$ gene.

strategies are required to improve the survival rate. The present case reports new findings: Positive expression of KIT and PDGFR $\alpha$ in the SmCC element and positive expression of EGFR and PDGFR $\alpha$ in the SqCC element. Furthermore, to the best of our knowledge, the present study is the first to report a KIT and PDGFR $\alpha$ mutation analysis in combined hypopharyngeal SmCC. KIT and PDGFR $\alpha$, both mapped to chromosome $4 \mathrm{q} 12$, have structural similarities with other $P D G F R$ family members. A majority of gastrointestinal stromal tumors (GISTs) display a gain-of-function mutation in the KIT proto-oncogene that encodes the KIT protein. The majority of mutations occur in KIT exon 11, but mutations 


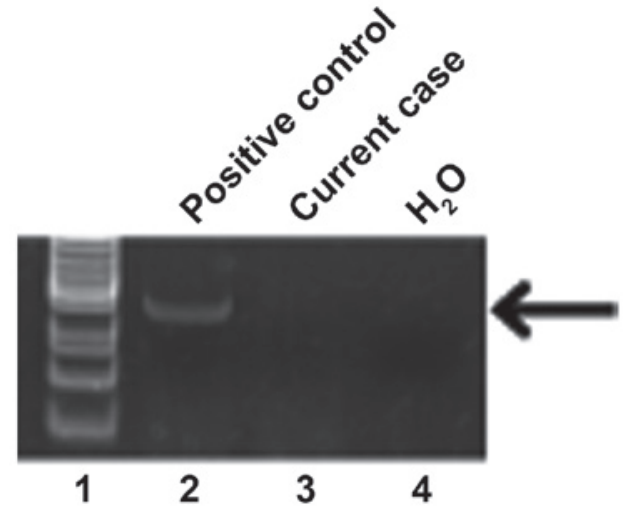

Figure 5. Electrophoresis of human papillomavirus (HPV) status. The band in lane 3 represents negative reactivity for HPV in this case, whereas lanes 2 and 4 correspond to the controls, which were the HPV-positive cell line UMSCC-47 (a gift from Dr TE Carey) and $\mathrm{H}_{2} \mathrm{O}$, respectively. The DNA size marker is located on lane 1.

may also be found in exon 9 and rarely in exons 13 and 17. The PDGFR $\alpha$ gene is also very similar to the KIT gene, and PDGFR $\alpha$ mutations have been found in exons 12, 14 and 18 . Terada et al (18) reported KIT protein expression in $100 \%$ and PDGFR $\alpha$ expression in $65 \%$ of small-cell lung carcinoma cases; however, there were no genetic mutations of KIT and $P D G F R \alpha$ in small-cell lung carcinoma (18). In a previously reported case of esophageal combined SmCC, KIT and PDGFR $\alpha$ were expressed in the SmCC component, but not in the SqCC component (5). It was found that SmCC of the lung and extrapulmonary organs expressed KIT and PDGFR $\alpha$, but there were no mutations in these genes within the hot spots of GISTs. These findings are almost completely in accord with those of our case study.

No biomarker has been yet proven to predict response to targeted therapy in either SmCC or SqCC. Moreover, a predictive biomarker in one cancer type may not be helpful in another cancer type, suggesting that different mechanisms may be involved in combined SmCC. To the best of our knowledge, a molecular-targeted therapy for treating combined SmCC of the hypopharynx and larynx has not yet been reported. Our case is the first to report KIT and PDGFR $\alpha$ expression in combined SmCC in the hypopharynx and larynx; if similar results are obtained from larger series, these data may have management and prognostic implications through the possible use of targeted biological therapy in these tumors. At present, SmCC treatment remains a significant challenge for the oncologists, with several targeted agents under evaluation. However, there is no effective molecular-targeted therapy for SmCC. Evaluating the expression of the KIT and PDGFR $\alpha$ genes within hypopharyngeal SmCC may facilitate the development of novel agents for the molecular-targeted therapy of this tumor. However, further investigations are necessary regarding these proteins expression and clinical application of molecular targeted therapy for hypophageal combined SmCC.

\section{Acknowledgements}

The authors would like to thank Ms. Yuko Mohri for her excellent technical support.

\section{References}

1. Ferlito A, Recher G and Caruso G: Primary combined small cell carcinoma of the larynx. Am J Otolaryngol 6: 302-308, 1985.

2. Mills SE, Cooper PH, Garland TA and Johns ME: Small cell undifferentiated carcinoma of the larynx. Report of two patients and review of 13 additional cases. Cancer 51: 116-120, 1983.

3. Uwa N, Terada T, Mohri T, Okazaki K, Tsukamoto Y, Hirota S and Sakagami M: Combined small cell carcinoma of the hypopharynx. Auris Nasus Larynx 40: 106-109, 2013.

4. Sobin LH, Wittekind C and Gospodarowicz M (eds): TNM Classification of Malignant Tumors. 7th edition. Wiley-Blackwell, New York, NY, 2009.

5. Terada T and Maruo H: Esophageal combined carcinomas: Immunohoistochemical and molecular genetic studies. World J Gastroenterol 18: 1545-1551, 2012.

6. Agaimy A, Wünsch $\mathrm{PH}$, Hofstaedter F, Blaszyk H, Rümmele $\mathrm{P}$, Gaumann A, Dietmaier W and Hartmann A: Minute gastric sclerosing stromal tumors (GIST tumorlets) are common in adults and frequently show c-KIT mutations. Am J Surg Pathol 31: 113-120, 2007.

7. Lasota $\mathbf{J}$ and Miettinen M: KIT and PDGFRA mutations in gastrointestinal stromal tumors (GISTs). Semin Diagn Pathol 23: 91-102, 2006.

8. Kikuchi H, Yamashita K, Kawabata T, Yamamoto M, Hiramatsu Y, Kondo K, Baba M, Ohta M, Kamiya K, Tanaka T, et al: Immunohistochemical and genetic features of gastric and metastatic liver gastrointestinal stromal tumors: Sequential analyses. Cancer Sci 97: 127-132, 2006.

9. Kartha RV and Sundram UN: Silent mutations in KIT and PDGFRA and coexpression of receptors with SCF and PDGFA in Merkel cell carcinoma: Implications for tyrosine kinase-based tumorigenesis. Mod Pathol 21: 96-104, 2008.

10. Renner G: Small cell carcinoma of the head and neck: A review. Semin Oncol 34: 3-14, 2007.

11. Mineta H, Miura K, Takebayashi S, Araki K, Ueda Y, Harada H and Misawa K: Immunohistochemical analysis of small cell carcinoma of the head and neck: A report of four patients and a review of sixteen patients in the literature with ectopic hormone production. Ann Otol Rhinol Laryngol 110: 76-82, 2001.

12. Barbeaux A, Duck L, Weynand B, Desuter G, Hamoir M, Gregoire V, Baurain JF and Machiels JP: Primary combined squamous and small cell carcinoma of the larynx: Report of two cases and discussion of treatment modalities. Eur Arch Otorhinolaryngol 263: 786-790, 2006.

13. Aggarwal G, Jackson L and Sharma S: Primary combined small cell carcinoma of larynx with lateralized histologic components and corresponding side-specific neck nodal metastasis: Report of a unique case and review of literature. Int J Clin Exp Pathol 4: 111-117, 2010.

14. Jaiswal VR and Hoang MP: Primary combined squamous and small cell carcinoma of the larynx: A case report and review of the literature. Arch Pathol Lab Med 128: 1279-1282, 2004.

15. Ferlito A, Silver CE, Bradford CR and Rinaldo A: Neuroendocrine neoplasms of the larynx: An overview. Head Neck 31: 1634-1646, 2009.

16. Barnes L, Eveson JW, Reichart P and Sidransky D: Pathology and Genetics. Head and Neck Tumours. World Health Organisation Classification of Tumours. IARC Press, Lyon, 2005.

17. Wu BZ, Gao Y and Yi B: Primary neuroendocrine carcinoma in oral cavity: Two case reports and review of the literature. J Oral Maxillofac Surg 72: 633-644, 2014.

18. Terada T: An immunohistochemical and molecular genetic analysis of KIT and PDGFRA in small cell lung carcinoma in Japanese. Int J Clin Exp Pathol 5: 331-338, 2012. 